\title{
A Double-Blind Multicenter Comparison of Domperidone and Metoclopramide in the Treatment of Diabetic Patients With Symptoms of Gastroparesis
}

\author{
David Patterson, M.D., Thomas Abell, M.D., Robin Rothstein, M.D., Kenneth Koch, M.D., \\ and Jeffrey Barnett, M.D. \\ Virginia Mason Clinic Hospital, Seattle, Washington; University of Tennessee Medical Center, Memphis, \\ Tennessee; Hospital of the University of Pennsylvania, Philadelphia, Pennsylvania; Milton S. Hershey \\ Medical Center, Hershey, Pennsylvania; and University of Michigan Medical Center, Ann Arbor, Michigan
}

OBJECTIVE: A double-blind, multicenter, randomized trial was conducted to compare the side effects and efficacy of domperidone and metoclopramide in symptomatic diabetic gastroparesis.

METHODS: Ninety-three insulin-dependent diabetes patients with a $\geq 3$-month history of gastroparesis symptoms were recruited; 48 received domperidone $2 \times 10-\mathrm{mg}$ tablets 4 times daily, and 45 received metoclopramide $1 \times 10-\mathrm{mg}$ tablet +1 placebo tablet 4 times daily. Nausea, vomiting, bloating/distension, and early satiety were evaluated for severity after 2 and 4 wk. Adverse central nervous system (CNS) effects of somnolence, akathisia, asthenia, anxiety, depression, and reduced mental acuity were elicited and graded for severity at 2 and 4 wk.

RESULTS: Domperidone and metoclopramide were equally effective in alleviating symptoms of diabetic gastroparesis. Elicited adverse CNS effects were more severe and more common with metoclopramide. Somnolence was acknowledged by $49 \%$ of patients (mean severity score, 1.03 ) after 4 wk of metoclopramide compared with $29 \%$ of patients (mean severity score, 0.49) after 4 wk of domperidone (incidence, $p=0.02$; severity; $p=0.03$ ). A reduction in mental acuity was acknowledged by $33 \%$ of patients (mean severity score, 0.62) after 4 wk of metoclopramide, compared with $20 \%$ of patients (mean severity score, 0.27 ) after 4 wk of domperidone (incidence, $p=0.04$; severity, $p=$ 0.04). Akathisia, asthenia, anxiety, and depression were also acknowledged less often, and at a lower severity, after $4 \mathrm{wk}$ of domperidone, although these differences were not statistically significant.

CONCLUSIONS: Domperidone and metoclopramide effectively reduce the symptoms of diabetic gastroparesis; CNS side effects are more pronounced with metoclopramide.

This study was prepared from the Janssen Research Foundation study report DOM-USA-001: Clinical evaluation of domperidone vs. metoclopramide in the treatment of diabetic patients with symptoms of gastroparesis-A multicenter study.
(Am J Gastroenterol 1999;94:1230-1234. (C) 1999 by Am. Coll. of Gastroenterology)

\section{INTRODUCTION}

Gastrointestinal motility disorders are common sequelae of diabetes mellitus, occurring in 10-30\%, and occasionally in as many as $75 \%$, of diabetic patients (1-3). Diabetic gastroparesis (DG), a syndrome characterized by symptoms of impaired gastric motility and delayed gastric emptying (4), may occur in both insulin-dependent (5) and non-insulindependent (6) diabetic patients. The symptoms of diabetic gastropathy, which may include postprandial nausea, epigastric burning or pain, bloating, vomiting of undigested food, anorexia, and early satiety, reduce the effectiveness of dietary regimens and the absorption of oral medications and, in general, make the underlying diabetes more difficult to control (5). In extreme cases of gastroparesis, nausea, vomiting, and bezoar formation may lead to an increased risk of hospitalization (7).

Although dietary measures, such as reducing the intake of solid foods in favor of liquefied meals, may diminish the risk of major complications in DG, they may significantly impair a patient's quality of life and general nutritional level. Effective drug therapy that allows for a normal diet may therefore offer a clear advantage over dietary measures in controlling DG.

Several prokinetic agents, including the dopamine $\mathrm{D}_{2}$ antagonists metoclopramide and domperidone, the cholinomimetic cisapride, and macrolide antibiotics such as erythromycin, have been used with varying degrees of success in the treatment of DG (8). However, only metoclopramide has been approved for the treatment of diabetic gastroparesis in the United States. Therapy with metoclopramide has been successful in relieving the symptoms of DG but is associated with prominent central nervous system (CNS) effects (drowsiness, restlessness, lassitude, and fatigue) in $10 \%$ of diabetic patients, and extrapyramidal reactions that may preclude its use $(8,9)$. Among patients with functional 
dyspepsia, the incidence of CNS-associated side effects (agitation, insomnia, somnolence, fatigue, and anxiety) was $23 \%$ in patients receiving metoclopramide therapy (10).

The efficacy of domperidone in the treatment of DG was demonstrated in a multicenter trial in insulin-dependent diabetic patients maintained on domperidone $20 \mathrm{mg} 4$ times daily for $4 \mathrm{wk}$. Patients given domperidone for $4 \mathrm{wk}$ had significant decreases $(p=0.001)$ in all symptoms of DG relative to baseline. Patients were then randomized to receive either domperidone for an additional $4 \mathrm{wk}$ or placebo; those who received domperidone had significantly $(p=$ $0.05)$ better symptom scores than patients switched to placebo. Other studies with domperidone have confirmed that its use results in shorter gastric emptying times and improved symptoms in patients with DG (11-13). Domperidone also produced a sustained improvement in gastric symptoms during long-term treatment of up to $1 \mathrm{yr}(14,15)$.

The ability of metoclopramide to cross the blood-brain barrier accounts for its CNS-associated adverse effects due to blockade of dopaminergic receptors. Domperidone does not readily cross the blood-brain barrier and would not be expected to interfere with central dopaminergic transmission $(16,17)$. Therefore, domperidone, a peripheral dopamine antagonist, would be expected to improve DG without inducing the CNS-associated side effects common with metoclopramide therapy.

The aim of the present trial was to compare the CNS tolerability profiles of domperidone and metoclopramide and to assess efficacy in patients with upper gastrointestinal symptoms suggestive of diabetic gastroparesis.

\section{PATIENTS AND METHODS}

Ambulatory male or female patients, aged $\geq 18$ years, with insulin-dependent diabetes mellitus and at least a 3-month history of symptoms of DG were eligible for inclusion in this multicenter (five centers), double-blind, randomized, parallel group design study. On study entry, patients were required to exhibit at least two of the following gastrointestinal symptoms: nausea, vomiting, bloating/distension, or early satiety. Investigators rated the severity of these four symptoms using a scale of 0 to 3 , where $0=$ none; $1=$ mild (present, but patient able to carry on usual activities); $2=$ moderate (interferes with activities); and $3=$ severe (disabling). The total symptom score (sum of the four individual gastrointestinal symptom scores) at study entry had to be at least 5 out of a possible score of 12 .

Patients with cancer of the gastrointestinal tract or major illnesses (end-stage heart, liver, or lung disease, alcoholism, cancer, or AIDS) were excluded from the study. Also excluded were patients who were receiving dialysis or who had undergone prior gastric surgery, those known or suspected to be using illicit drugs, and those who had received either study drug or an investigational drug within 30 days before study entry. Pregnant women and those likely to become pregnant during the study were also excluded, but women using adequate contraception were allowed in the trial. Each patient signed a written informed consent statement before entry into the study. Institutional review board approval was obtained before the start of the study.

On study entry, patients provided a medical history and underwent a physical examination, including laboratory determinations, hematology, blood chemistry, and urinalysis. Patients who met entry criteria received either domperidone in the dosage shown to be effective in previous clinical studies of diabetic gastroparesis $(11,14,15), 20 \mathrm{mg} 4$ times daily $(\mathrm{n}=48)$, or the recommended dosage of metoclopramide for the treatment of diabetic gastroparesis, $10 \mathrm{mg} 4$ times daily $(\mathrm{n}=47)$, for $4 \mathrm{wk}$. Patients received domperidone (two 10-mg tablets) or metoclopramide (one 10-mg tablet and one placebo tablet) 15-30 min before meals (breakfast, lunch, and dinner) and at bedtime.

Medications that could mask the effect of domperidone and metoclopramide (e.g., cisapride or bethanechol) were not permitted during the study. Use of anticholinergics, neuroleptics, opiates, significant analgesics, antiemetics, histamine $\mathrm{H}_{2}$-receptor antagonists, sucralfate, and omeprazole was discouraged where possible. If antacids or histamine $\mathrm{H}_{2}$-receptor antagonists were required, they were not to be taken within 30 min of ingestion of the study drug.

Patients were evaluated at Weeks 2 and 4 to evaluate symptoms, note any spontaneously reported adverse experiences, and assess patient compliance (on the basis of unused tablet counts). The patients were also asked specifically if they had experienced any of the CNS-associated side effects that are most common during therapy with metoclopramide (somnolence, akathisia, asthenia, anxiety, depression, or reduced mental acuity), and to grade the severity of these occurrences on the symptom severity scale described above. These elicited CNS-associated adverse effects served as primary indicators of the relative tolerability of the two agents. The primary measures of drug efficacy were the symptom scores for nausea, vomiting, early satiety, and bloating/distention. Repeat physical examination and laboratory tests (hematology, blood chemistry, and urinalysis) were performed on study completion.

Demographic data and baseline vital signs for each treatment group were compared using Student's $t$ test. Noncontinuous variables (sex, race, and global assessments) were compared using the Cochran-Mantel-Haenzel test. Between-treatment comparisons for baseline, 2-wk, and 4-wk means were performed using a two-way ANOVA for the symptom and elicited adverse event severities. Treatment, investigator, and treatment-by-investigator interactions were evaluated. Within-treatment comparisons were performed using a Student's paired $t$ test. For the elicited adverse event data, between-group comparisons of incidences were performed using the Cochran-Mantel-Haenzel test. Symptom and adverse effect severity scores were confirmed nonparametrically using the Wilcoxon signed-rank test for within-group comparisons and two-way ANOVA of the ranked data for between-group comparisons. All com- 


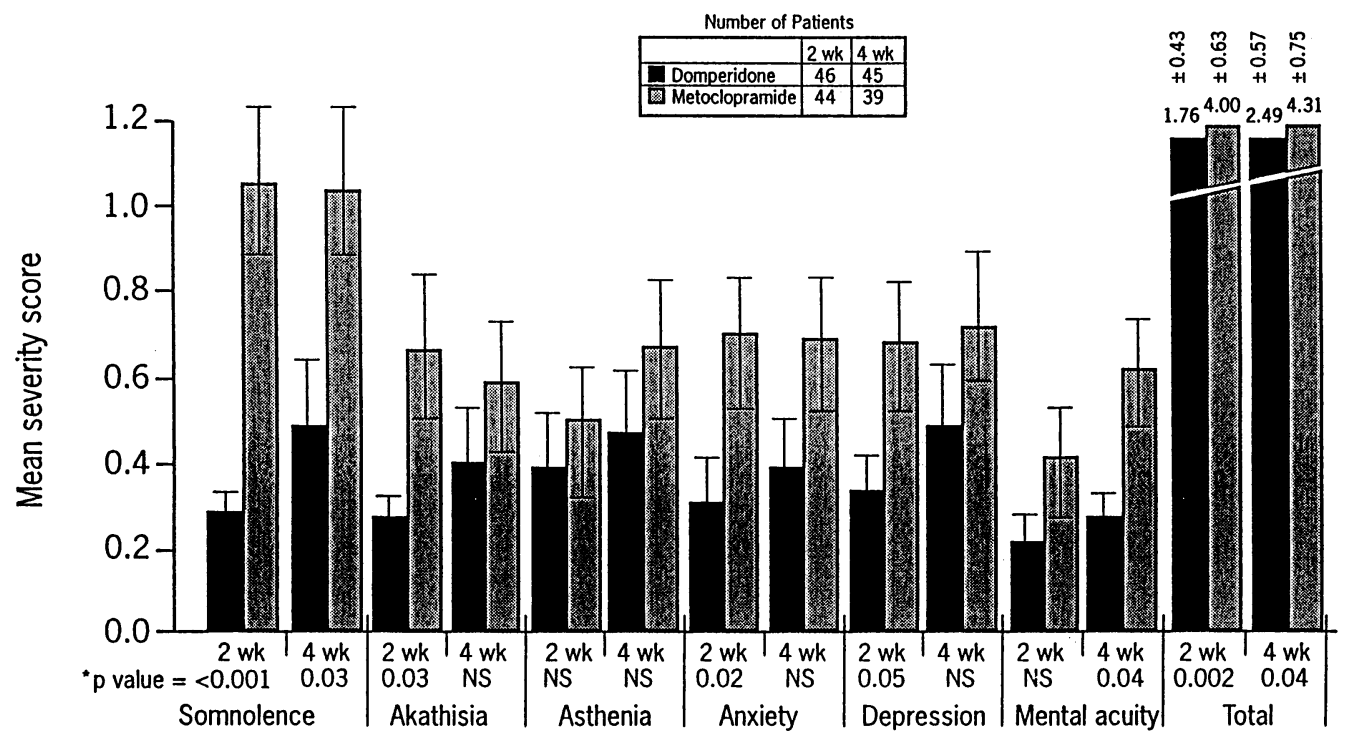

Figure 1. Severity of elicited CNS-associated adverse events in diabetic patients treated with either domperidone or metoclopramide. The total score is the sum of the six individual scores. *Two-sided $p$ value, with domperidone having the lower score.

parisons were two-tailed, with a probability value $\leq 0.05$ signifying statistical significance.

\section{RESULTS}

\section{Patients}

Of the 95 patients who entered the study and were randomized to double-blind treatment, 33 were male and 62 were female. The majority of patients were white $(80.0 \%)$, nonsmokers $(76.8 \%)$, and nondrinkers $(77.9 \%)$, and were receiving concomitant insulin therapy $(99.0 \%)$. Patients ranged in age from 19 to 69 years (median, 39 years), in weight from 41 to $122 \mathrm{~kg}$ (median, $68.2 \mathrm{~kg}$ ), and in height from 1.47 to $1.96 \mathrm{~m}$ (median, $1.68 \mathrm{~m}$ ). The two treatment groups were comparable with respect to demographics, medical backgrounds, vital signs, and severity of DG symptoms at baseline. Two patients in the metoclopramide group failed to provide efficacy data; therefore, intent-to-treat analysis involved a total of 93 patients ( 48 domperidone and 45 metoclopramide recipients). Of these, 16 patients (six domperidone and 10 metoclopramide recipients) discontinued treatment prematurely.

\section{Tolerability}

Elicited adverse CNS effects were more severe during metoclopramide therapy than during domperidone therapy. At 2 wk, the severities of somnolence, akathisia, anxiety, and depression were significantly greater $(p<0.001-0.05$; see Fig. 1) with metoclopramide than with domperidone. At 4 wk, the severities of somnolence and reduced mental acuity

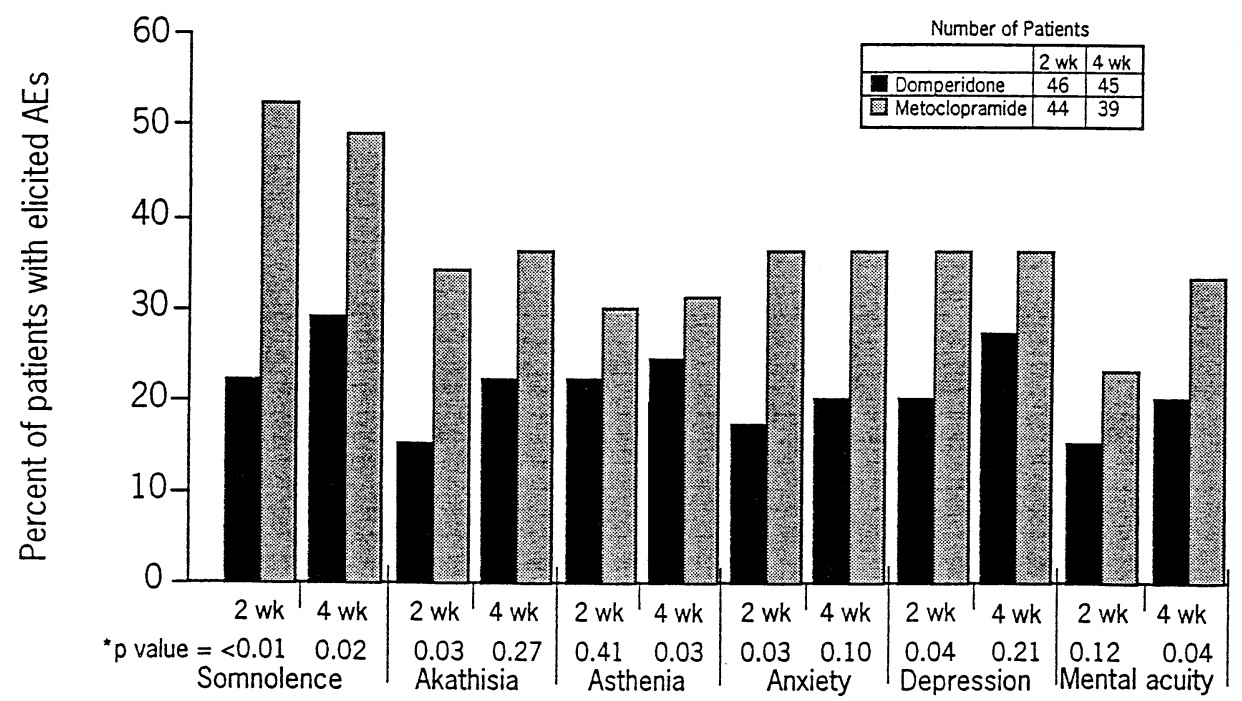

Figure 2. Incidence of elicited CNS-associated adverse events in diabetic patients treated with either domperidone or metoclopramide. *Cochran-Mantel-Haenzel test, with domperidone having the lower incidence. NS = not significant. 
Table 1. Incidences of the Most Frequent Spontaneously Reported Adverse Events Occurring With Domperidone and Metoclopramide

\begin{tabular}{lcc}
\hline Adverse Event & $\begin{array}{c}\text { Domperidone } \\
(\mathrm{n}=48)\end{array}$ & $\begin{array}{c}\text { Metoclopramide } \\
(\mathrm{n}=47)\end{array}$ \\
Vomiting & 10.4 & 4.3 \\
Nausea & 10.4 & 4.3 \\
Headache & 6.3 & 2.1 \\
Insomnia & 6.3 & 0 \\
Diarrhea & 6.3 & 2.1 \\
Prolactin-related & 6.3 & 6.4 \\
Cystitis & 6.3 & 2.1 \\
\hline
\end{tabular}

were significantly greater $(p=0.03-0.04$; see Fig. 1$)$ with metoclopramide than with domperidone.

Elicited adverse CNS effects occurred more frequently during metoclopramide therapy than during domperidone therapy. At 2 wk, the incidences of somnolence, akathisia, anxiety, and depression were significantly greater $(p<$ 0.01-0.04; see Fig. 2) with metoclopramide than with domperidone. At $4 \mathrm{wk}$, the incidences of somnolence and reduced mental acuity were significantly greater $(p=0.02-$ 0.04 ; see Fig. 2) with metoclopramide than with domperidone.

Of the spontaneously reported adverse effects, nausea, vomiting, headache, insomnia, and diarrhea occurred in $6-10 \%$ of domperidone-treated patients and in up to $4 \%$ of metoclopramide recipients. The incidence of prolactin-related adverse effects was similar $(\approx 6 \%)$ in the two treatment groups (Table 1).

A total of nine patients (three domperidone recipients and six metoclopramide recipients) discontinued therapy because of adverse events. Elicited adverse CNS effects were responsible for treatment discontinuation in four metoclopramide-treated patients and in one domperidone-treated patient. No clinically relevant changes in laboratory parameters were evident during treatment with either domperidone or metoclopramide.

\section{Therapeutic Efficacy}

Both treatment groups showed significant reductions in the severity of gastroparetic symptoms (Fig. 3). From baseline to endpoint, the total symptom score (sum of four individual symptom scores: nausea, vomiting, early satiety, and bloating/distension) fell from $8.0 \pm 0.32$ to $4.71 \pm 0.46(41.1 \%$ reduction) with domperidone and from $8.33 \pm 0.29$ to $5.09 \pm 0.5$ (38.9\% reduction) with metoclopramide $(p=$ NS). No significant difference was noted between the effects of the two treatments on any gastrointestinal symptom.

\section{DISCUSSION}

In the present study, treatment with domperidone $20 \mathrm{mg} 4$ times daily or metoclopramide $10 \mathrm{mg} 4$ times daily for $4 \mathrm{wk}$ were similarly effective in improving gastrointestinal symptoms suggestive of DG in patients with insulin-dependent

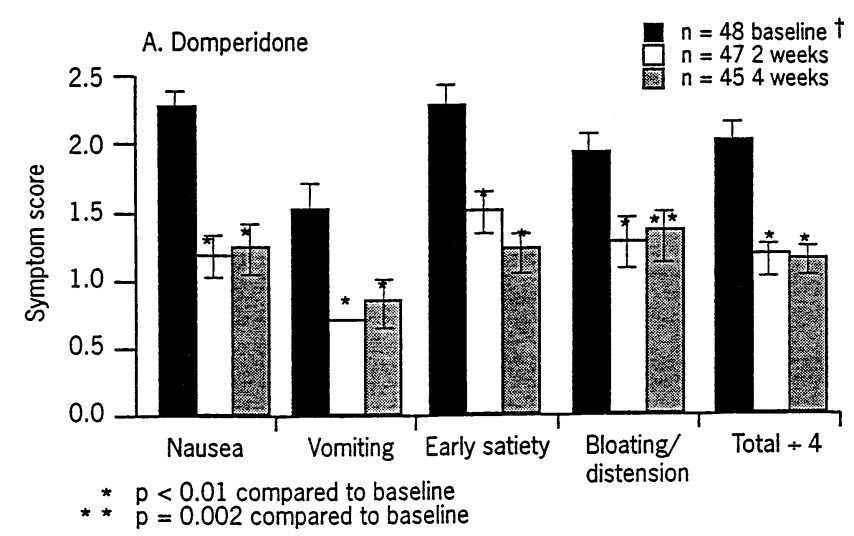

Figure 3. Gastroparetic symptom scores after either domperidone or metoclopramide in patients with insulin-dependent diabetes mellitus. There were no significant differences between treatment groups for any individual or total symptom assessments, or for any timepoint.

diabetes. These results are consistent with the findings of other studies in which domperidone or metoclopramide administered chronically demonstrated significant improvement in symptoms of DG, compared with placebo $(11,12$, 18).

When patients were questioned specifically, it was found that both the incidence and severity of CNS adverse events common to metoclopramide were lower in patients receiving domperidone than in patients receiving metoclopramide. These results are consistent with the findings of a previously published double-blind, placebo-controlled crossover study of domperidone and metoclopramide, in which 11 patients receiving metoclopramide reported side effects (including dizziness, depression, and lethargy), as compared with only two patients receiving domperidone and three patients receiving placebo (19). This observed difference is probably due to the ability of metoclopramide to cross the blood-brain barrier and the limited ability of domperidone to cross the blood-brain barrier $(16,17)$.

In conclusion, domperidone $20 \mathrm{mg} 4$ times daily and metoclopramide $10 \mathrm{mg} 4$ times daily appear to have similar efficacy in reducing the gastrointestinal symptoms of DG, although domperidone offers a superior tolerability in terms of unwanted CNS effects.

\section{ACKNOWLEDGMENTS}

This study was funded in part by Janssen Research Foundation, Titusville, New Jersey.

Reprint requests and correspondence: David Patterson, M.D., Virginia Mason Clinic, 1100 Ninth Avenue, Seattle, WA 98101. Received July 19, 1995; accepted May 28, 1998.

\section{REFERENCES}

1. Feldman M, Schiller LR. Disorders of gastrointestinal motility associated with diabetes mellitus. Ann Intern Med 1983;98: $378-84$. 
2. Keshavarzian A, Iber FL. Gastrointestinal involvement in insulin-requiring diabetes mellitus. J Clin Gastroenterol 1987; 9:685-92.

3. Van Dijl PW, Hoffman SJ, Van Doormaal JJ, et al. Low prevalence of symptomatic gastric and esophageal motor dysfunction in diabetes mellitus. Gastroenterology 1989;96:A524.

4. Reynolds JC. Prokinetic agents: A key in the future of gastroenterology. Gastroenterol Clinics North Am 1989;18:43757.

5. Kassander P. Asymptomatic gastric retention in diabetics (gastroparesis diabetoricum). Ann Int Med 1958;48:797-812.

6. Horowitz M, Harding PE, Maddox AF, et al. Gastric and oesophageal emptying in patients with Type 2 (non-insulindependent) diabetes mellitus. Diabetologia 1989;32:151-9.

7. von der Ohe M, Camilleri M, Zimmerman BR. Management of diabetic enteropathy. The Endocrinologist 1993;3:400-8.

8. Drenth JPH, Engels LGJB. Diabetic gastroparesis. A critical reappraisal of new treatment strategies. Drugs 1992;44:53753.

9. Albibi R, McCallum RW. Metoclopramide: Pharmacology and clinical application. Ann Intern Med 1983;98:86-95.

10. Van Outryve M, De Nutte N, Van Eghem P, et al. Efficacy of cisapride in functional dyspepsia resistant to domperidone or metoclopramide: A double-blind, placebo-controlled study. Scand J Gastroenterol 1993;28(suppl 195):47-53.

11. Champion MC, Gulenchyn K, O'Leary T, et al. Domperidone (Motilium) improves symptoms and solid phase gastric emp- tying in diabetic gastroparesis. Am J Gastroenterol 1987;82: 975.

12. Heer M, Muller-Duysing W, Benes I, et al. Diabetic gastroparesis: Treatment with domperidone-A double-blind, placebo-controlled trial. Digestion 1983;27:214-7.

13. Horowitz M, Harding PE, Chatterton BE, et al. Acute and chronic effects of domperidone on gastric emptying in diabetic autonomic neuropathy. Dig Dis Sci 1985;30:1-9.

14. Koch KL, Stern RM, Stewart WR, et al. Gastric emptying and gastric myoelectrical activity in patients with diabetic gastroparesis: Effect of long-term domperidone treatment. Am J Gastroenterol 1989;84:1069-75.

15. Kozarek R. Domperidone for symptomatic management of diabetic gastroparesis in metoclopramide treatment failures. Adv Ther 1990;7:61-8.

16. McCallum RW: Review of the current status of prokinetic agents in gastroenterology. Am J Gastroenterol 1985;80: 1008-16.

17. Parkes JD. Domperidone and Parkinson's disease. Clin Neuropharmacol 1986;9:517-31

18. Ahn YH, Maturu P, Steinheber FU, Goldman JM. Association of diabetes mellitus with gastric bezoar formation. Arch Intern Med 1987;147:527-8.

19. Maddern GJ, Kiroff GK, Leppard PI, et al. Domperidone, metoclopramide, and placebo. J Clin Gastroenterol 1986;8: 135-40. 